\title{
Exploration of attractor modules for sporadic amyotrophic lateral sclerosis via systemic module inference and attract method
}

\author{
FANG ZHANG ${ }^{1}$, MEI LIU ${ }^{1}, \mathrm{QUN} \mathrm{LI}^{1}$ and FEI-XUE SONG ${ }^{2}$ \\ Departments of ${ }^{1}$ Rehabilitation and ${ }^{2}$ Oncology, The Second Hospital of Lanzhou University, \\ Lanzhou, Gansu 730030, P.R. China
}

Received May 22, 2018; Accepted February 1, 2019

DOI: $10.3892 /$ etm.2019.7264

\begin{abstract}
Sporadic amyotrophic lateral sclerosis (SALS) is a devastating neurodegenerative disorder. However, the understanding of SALS is still poor. This research aimed to excavate attractor modules for SALS by integrating the systemic module inference and attract method. To achieve this, gene expression data and protein-protein data were recruited and preprocessed. Then, based on the Spearman's correlation coefficient (SCC) of the interactions under these two conditions, two PPI networks separately with 870 nodes (979 interactions) in normal control group and 601 nodes (777 interactions) in SALS group were built. Systemic module inference method was performed to identify the modules, and attract method was used to identify attractor modules. Finally, pathway enrichment analysis was performed to disclose the functional enrichment of these attractor modules. In total 44 and 118 modules were identified for normal control and SALS groups, respectively. Among them, 6 modules were with similar gene composition between the two groups, and all 6 modules were considered as the attractor module via attract method. These attractor modules might be potential biomarkers for early diagnosis and therapy of SALS, which could provide insight into the disease biology and suggest possible directions for drug screening programs.
\end{abstract}

\section{Introduction}

Amyotrophic lateral sclerosis (ALS) is characterized by widespread loss of motor neurons in the major motor cortex and is a devastating neurodegenerative disorder (1). Approximately $10 \%$ of ALS cases have a family history and the other $90 \%$ of cases are sporadic amyotrophic lateral sclerosis (SALS) $(2,3)$. To date, $>150$ genetic mutations and $>25$ different genes have been identified to possibly lead to

Correspondence to: Dr Fei-Xue Song, Department of Oncology, The Second Hospital of Lanzhou University, 82 Cuiyingmen, Chengguan, Lanzhou, Gansu 730030, P.R. China

E-mail: feixue_song@163.com

Key words: sporadic amyotrophic lateral sclerosis, proteinprotein interaction network, attractor module, pathway, Spearman's correlation coefficient the same clinical disease of FALS in patients, and it is characterized to some extent (4). However, the understanding for the more common SALS is still poor and there is no effective treatment or definitive diagnostic test for SALS (5). Identification of genetic factors may provide some insight into the underlying mechanisms of this motor neuron degeneration disease (6). Recently, the mutations of PFN1 (7), CAMTA1 (8), and MATR3 (9) have been identified to be associated with SALS cases. Moreover, the whole gene composition of the SALS has been identified (10). Even though researchers have tried their best to overcome this complicated and refractory disease, the pathological mechanism of SALS is still unclear.

During the past several years, high-throughput experimental and the second generation sequencing technologies have produced large amounts of gene expression profile data, and make it possible to study the pathological mechanism of a certain disease systematically (11). It is well known that the genes that are functionally related to the disease are always highly co-expressed across organisms (12). In other words, they are active in modules (13). Fortunately, several module approaches have been proposed to determine the differential expression of the modules under differential cell types, and systemic module inference method (14) and attract method (15) is the most frequently used methods to conducted analysis on the differential expressed modules. The systemic module inference method can reveal the interesting patterns between gene composition and expression correlation, especially those affecting modules for genomic stability (14), and the attract method can best discriminate the differences between different cell phenotypes, and is not restricted to the annotated genes (15).

Therefore, to understand the mechanism of SALS, we integrated the systemic module inference method and the attract method to conduct analysis on the gene expression of SALS, so as to identify the differentially expressed modules, which we called attractor modules for SALS. The results might provide insight into potential biomarkers for early diagnosis and therapy of SALS, or even give a hand for clinical treatment of this complicated and refractory disease and other related diseases.

\section{Materials and methods}

Gene expression data recruiting and preprocessing. Data recruitment of SALS, with accession number E-MTAB-2325, 
was conducted from ArrayExpress database (http://www.ebi. ac.uk/arrayexpress/). E-MTAB-2325 exists on A-AGIL-28 Agilent Whole Human Genome Microarray 4x44K 014850 G4112F ( 85 cols $x$ 532 rows) Platform, and consist of 41 samples (10 normal control samples and 31 SALC patients).

Prior to analysis, the robust multichip average (RMA) method (16) was used to perform background correction and quantile based algorithm (17) and invited to carry out normalization to debride the effect of nonspecific hybridization. Besides, Micro Array Suite 5.0 (MAS 5.0) algorithm was used to amend perfect match and mismatch value (18). Moreover, the median polish was utilized to conduct the calculation of the gene expression value analysis (19). Finally, all the genes in the probe level were converted into gene symbols, and 18,411 gene symbols were procured.

PPI network construction. To construct the PPI network, data were firstly recruited from the database Search Tool for the Retrieval of Interacting Genes/Proteins (STRING, http://string-db.org/). The data comprised of 1,048,576 interactions with combine-scores obtained from STRING in order to build PPI network. Following the removing of self-loops and proteins without expression value, a PPI network containing 7,279 nodes and 43,786 highly correlated interactions (with combine-score $\geq 0.7$ ) was constructed. Taking the intersection of the 12,493 genes in E-MTAB-2325 and the nodes in the PPI network, we established a novel PPI network with 7,033 nodes (43,786 interactions).

PPI network re-weighting. The score of an interaction could represent the reliability of PPI network, and the re-weighted interaction could better reflect the practical network interaction than the original one (20). In the present study, PPI networks under two conditions were separately re-weighted by the parameter of Spearman's correlation coefficient (SCC) to reflect the actual relationship between these interactions. First of all, the SCC of each interaction in the novel PPI network was calculated according to the gene expression profile value, and the absolute values of the SCC were separately considered as the combine-score value of each interaction. Then, a two-tailed t-test with Benjamini-Hochberg false discovery rate (FDR) adjustment was used to identify the P-values of differential gene expression between SALC and control conditions (21), and the interactions with P-value $<0.05$ were established to build the destination networks for normal control and SALS groups, respectively. In the circumstances, two PPI networks separately with each of the edges were assigned a combined score were built for normal control and the SALS groups, respectively.

Identifying maximal cliques from the PPI networks. A maximal clique is a maximal independent set of sub-graph in a graph, and any two vertices in the clique are adjacent (22). The maximal cliques are always one of the fundamental problems in a certain network, and identifying maximal cliques has been widely used in bioinformatics and clustering (23). Here, for identifying the maximal cliques from the PPI networks, we invited the fast depth-first method to perform the analysis. All of the maximal cliques were ranked according to the number of nodes. The cliques with too small amount of nodes might be too simple and insufficient to describe the correlation of the biomarkers and the disease while the cliques with too large numbers of genes were not easy enough to be understood by human experts. Hence, we only retained the maximal cliques whose number of nodes was not less than 6 and not larger than 20 for further analysis in this study.

Identifying modules. Furthermore, to identify modules for normal control and the SALC groups, clique-merging were utilized to perform module-identification algorithm in this study (24). First of all, the weighted interaction density (WID) was separately calculated for each maximal cliques that we identified above, and all of the maximal cliques were ranked in descending order on the basis of their values of WID. Moreover, there might be thousands of maximal cliques in a PPI network and most of them overlapped with one another, and the highly overlapped cliques should be removed to reduce the result size. Moreover, merging highly overlapped cliques to form bigger sub-graphs was also desirable since complexes were not necessarily fully connected and PPI data might be incomplete. Hence, the inter-connectivity between any two cliques was calculated according to the WID values, and the inter-connectivity values were used to determine whether two overlapped cliques should be merged together or not. In the present study, the inter-connectivity value $>0.5$ was used as the cut off value whether to merge or not merge these two maximal cliques.

Comparison of the genes in the modules under different conditions. In order to better compare the differential expression of the modules between normal control and the SALS conditions, the modules that were with same or similar genetic make-up in the two groups were determined. The Jaccard similarity of the module in the case and control condition were identified, which was calculated according to $J\left(S_{a}, T_{b}\right)=\left|S_{a} \cap T_{b}\right| /\left|S_{a} \cup T_{b}\right|(25)$. The modules $J\left(S_{a}, T_{b}\right)$ $\geq 0.7$ were considered as similar modules in gene composition. In the present study, all of the modules in similar gene composition were considered as candidate attractors in the following analysis.

Identification of attractor modules. Further to determine the differential attractor modules between the normal control and the SALS groups, the attract method was utilized to perform analysis on the candidate attractor. In the present study, GSEA-ANOVA, a gene set enrichment algorithm was used to indentify the differential expression on the attractor level data.

First of all, take gene $m$ as an example, an ANOVA model was fit to it as its gene expression was modeled by a single factor. Suppose that there was $\mathrm{u}(\mathrm{u}=1, \ldots, \mathrm{u})$ samples and $\mathrm{v}$ $(\mathrm{v}=1, \ldots, \mathrm{v})$ cell phenotypes, the gene expression profile of gene $m$ was modeled in light of the following formula:

$$
y_{u_{v}}^{(m)}=\beta+\beta_{\mathrm{v}}+\delta_{u_{v}}
$$

$\beta$ represented the overall mean, $\beta$ denoted the $u$-th cell type group's effect on the expression of the gene $m$, and $\delta_{u_{v}}$ reflected the random normal residual error term. 


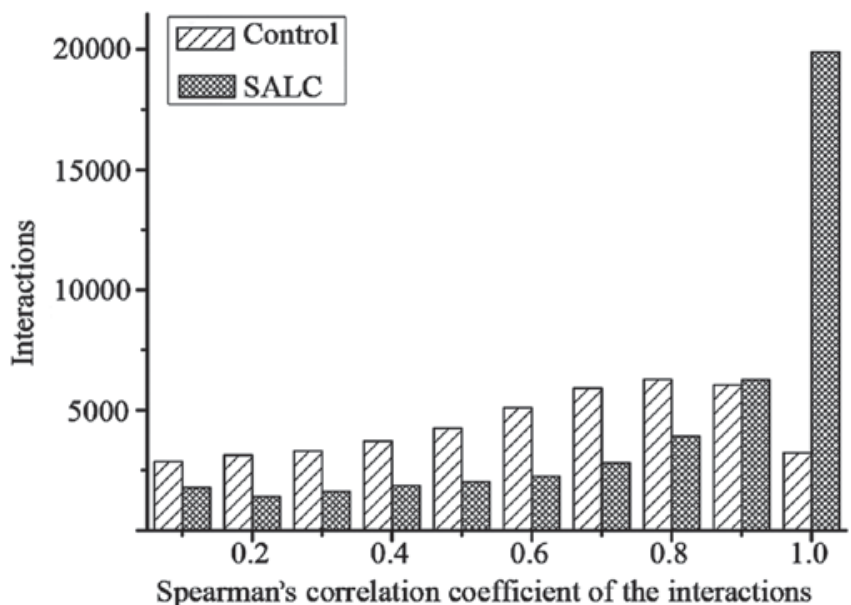

Figure 1. The distributions of the absolute value of Spearman's correlation coefficient (SCC) in the normal control and the sporadic amyotrophic lateral sclerosis groups. The X-axis denotes the absolute value of the SCC, and the Y-axis represents the number of interactions.

Then, an F-statistic value was assigned to gene $m$, which was calculated in light of the following formula:

$$
F^{(m)}=\frac{M S S_{m}}{R S S_{m}}
$$

$M S S_{a}$ represents the mean treatment sum of squares, which was determined by the amount of variation according to the cell type group-specific effects, and $R S S_{a}$ denotes the residual sum of squares.

In this case, we could identify which genes were informative for a particular set of cell types by integrating the ANOVA model with the F-statistics. As it was indicated that the F-statistic could capture the strength of association of a gene's expression over the different cell types, and the larger the F-statistic values were, the larger the cell type-specific expression changes (26). Therefore, in the present study, to test the relationship between the F-statistic values and cell type-specific expression changes, we used t-test and Welch modification to perform further analysis. For increasing the sensitivity of the differences between the global distribution of F-statistics and the module distribution, we performed a multiple-testing by using the Benjamini-Hochberg FDR-based method to adjust the resulting P-values (27). Finally, these candidate attractors with adjusted P-values $<0.05$ were regarded as attractor modules.

Pathway enrichment analysis of the attractor modules. To determine the functional enrichment of these attractor modules, pathway enrichment analysis was grounded in Kyoto Encyclopedia of Genes and Genomes (KEGG) pathway database in Genelibs (http://www.genelibs.com/gb/index.jsp). To determine the P-values of the enrichment condition, the Fisher's exact test was performed. Moreover, Benjamini-Hochberg method was utilized to go on multiple testing on the P-values. The pathways with adjusted P-value $<0.05$ were considered as the pathways where certain attractor module was enriched. Additionally, the pathway with the minimum adjusted P-value was considered as the significant pathway that the attractor module was enriched in.

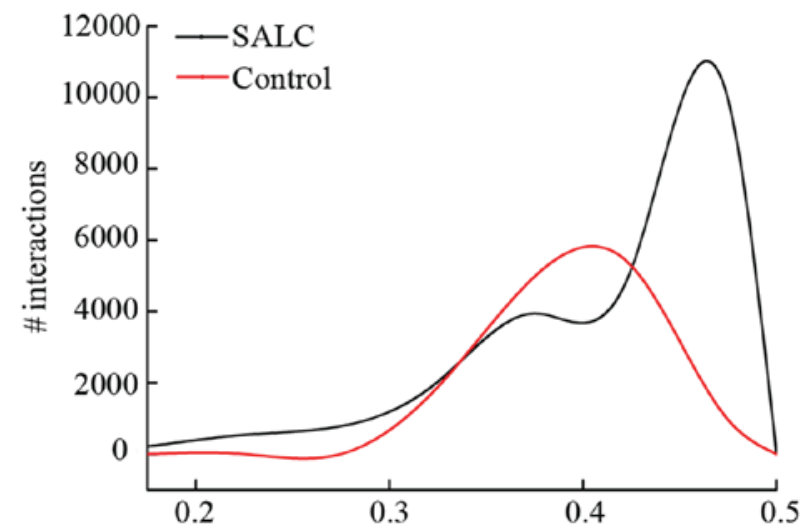

Weighted interaction density (WID) of the maximal cliques

Figure 2. The distributions of the weighted interaction density in the normal control and the sporadic amyotrophic lateral sclerosis groups. The $\mathrm{X}$-axis denotes the WID values, and the Y-axis represents the number of interactions.

\section{Results}

PPI network re-weighting. To present the reliability of the network, the interactions were all re-weighted to reflect an actual interaction network based on SCC. As statistical analysis was conducted on the distributions of the absolute value of SCC in the normal control and the SALS groups, we found that the distributions were different from each other (Fig. 1). To increase the comparability of these two groups, a twotailed t-test with Benjamini \& Hochberg FDR adjustment was utilized to determine the P-values of differential gene expression between the normal control and the SALS conditions, and under the cut-off value of P-value $<0.05$, two PPI networks separately with each of the edges were assigned a combined-score built for the normal control and the SALS groups, respectively. However, there were some nodes out of the main networks, and these nodes with lower degree were not associated with the biological process of a certain disease. Hence, to conduct the following analysis more conveniently, the nodes out of the main networks were removed, and only the main networks remained to perform a further analysis. There were 870 nodes (979 interactions) in the normal control group and 601 nodes (777 interactions) in the SALS group of the main PPI networks, respectively.

Identifying maximal cliques from the PPI networks. Maximal cliques were one of the fundamental problems in a certain network, hence in the present work, we identified the maximal cliques to perform analysis on the reweighted network. The fast depth-first method identified the maximal cliques from the PPI networks, and we gained 12,849 and 22,605 maximal cliques, respectively, for the normal control and the SALS groups. Finally, under the threshold value of node number larger than 6 and less than 20,1,474 and 4,224 maximal cliques, respectively, were obtained for the normal control and the SALS groups for further analysis.

Identifying modules. To further refine the maximal cliques, modules were identified based on the clique-merging. After the WID values in both groups were calculated and ranked in 


\section{Module 1}

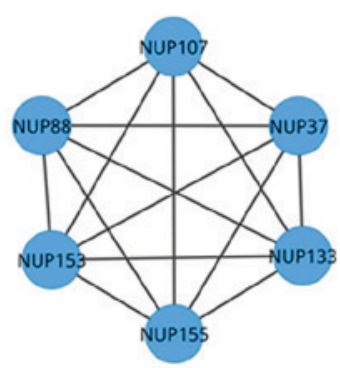

Module 4

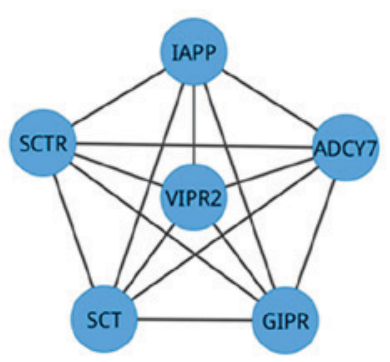

Module 2

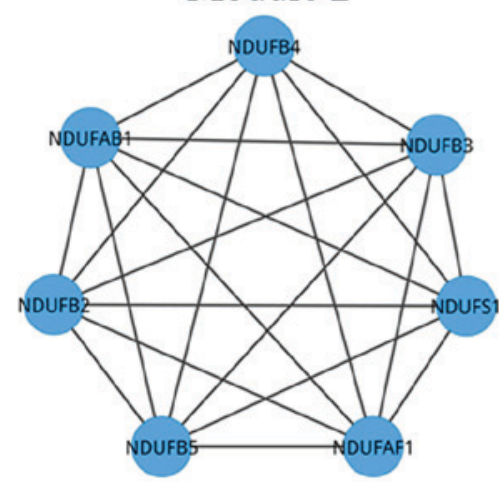

Module 5

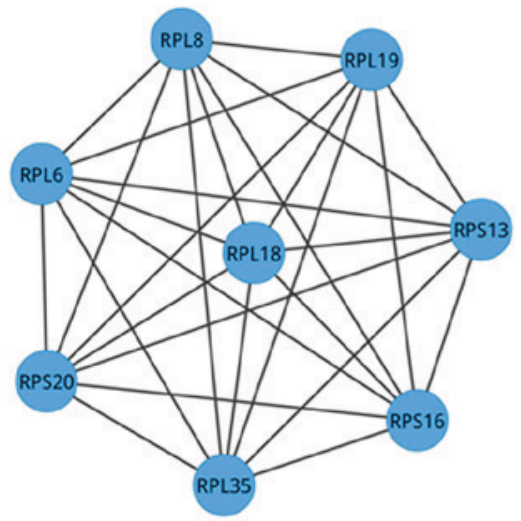

Module 3

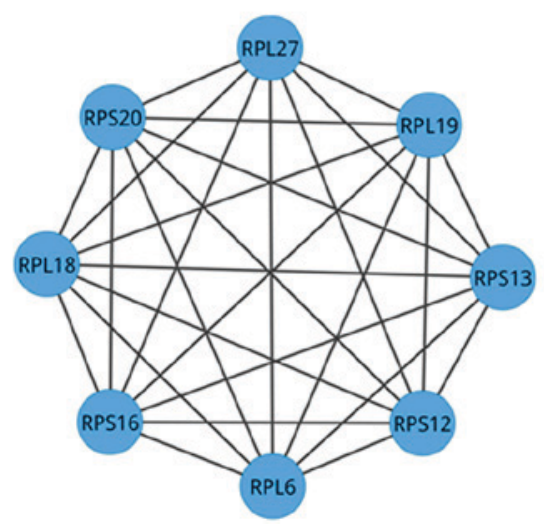

Module 6

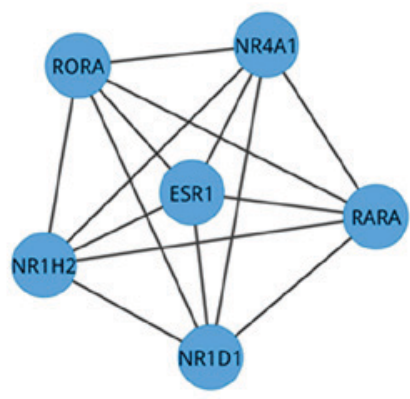

Figure 3. The 6 attractor modules identified based on the approach of integrating the systemic module inference method with the attract method. The yellow nodes represent the genes of the modules and the lines denote the interactions between these genes.

Table I. Details of the attractor modules and the pathway.

\begin{tabular}{|c|c|c|c|c|}
\hline Module & P-value & $\begin{array}{l}\text { Gene } \\
\text { number }\end{array}$ & Gene & $\begin{array}{l}\text { Enriched pathway } \\
\quad \text { (P-value) }\end{array}$ \\
\hline Module 1 & 0.3130570 & 6 & NUP88, NUP107, NUP37, NUP153, NUP133, NUP155 & NA \\
\hline Module 2 & 0.2126378 & 7 & $\begin{array}{l}\text { NDUFAB1, NDUFB2, NDUFB4, NDUFB5, } \\
\text { NDUFB3, NDUFS1, NDUFAF1 }\end{array}$ & $\begin{array}{l}\text { Oxidative phosphorylation } \\
\left(\mathrm{P}=1.63 \times 10^{-10}\right)\end{array}$ \\
\hline Module 3 & 0.4126378 & 8 & $\begin{array}{l}\text { RPS20, RPL18, RPS16, RPL6, RPS13, RPL27, } \\
\text { RPS12, RPL19 }\end{array}$ & $\begin{array}{l}\text { EIF2 signaling } \\
\left(\mathrm{P}=3.67 \times 10^{-12}\right)\end{array}$ \\
\hline Module 4 & 0.4126378 & 6 & SCTR, SCT, IAPP, ADCY7, GIPR, VIPR2 & $\begin{array}{l}\text { Neuroactive ligand-receptor } \\
\text { interaction }\left(\mathrm{P}=1.11 \times 10^{-3}\right)\end{array}$ \\
\hline Module 5 & 0.5841532 & 8 & $\begin{array}{l}\text { RPS20, RPL35, RPL18, RPS16, RPL6, RPS13, } \\
\text { RPL8, RPL19 }\end{array}$ & NA \\
\hline Module 6 & 0.5841532 & 6 & ESR1, NR4A1, RARA, NR1H2, NR1D1, RORA & $\begin{array}{l}\text { Circadian rhythm } \\
\left(\mathrm{P}=0.2 .31 \times 10^{-3}\right)\end{array}$ \\
\hline
\end{tabular}

descending order, we found that the WID values were ranged from 0.316 to 0.498 in the normal control group, and in the SALS group they ranged from 0.178 to 0.499 (Fig. 2). Thus, the cliques in the normal control group were more likely to have more common neighbours than the cliques in the SALS group. Moreover, the inter-connectivity between two cliques was defined based on the connectivity between the non-overlapping parts of the two cliques. Under the threshold value of the inter-connectivity value $>0.5,44$ and 118 modules were confirmed as the normal control and the SALS groups, respectively.

Comparison of the genes in the modules under different condition. Having identified modules for both groups, for better comparing the differential modules between normal control and the SALS conditions, we performed analysis 
to determine the modules that were with same or similar genetic make-up in the two groups. Under the cut-off value of $J\left(S_{a}, T_{b}\right) \geq 0.7,6$ candidate attractors, which we named as module 1 - module 6 were selected for further analysis.

Identification of attractor modules. To determine the differential expression normal control and the SALS groups, the attract method was conducted for analysis on the attractor level. After having used the t-test and Welch modification to determine the F-statistic values and cell type-specific expression changes, the resulting P-values were adjusted by using Benjamini-Hochberg FDR-based method, 6 attractor modules were identified under the threshold value of adjusted P-values $<0.05$. As shown in Fig. 3, in module 1 to module 6, there were, respectively, 6 nodes (15 interactions), 7 nodes (21 interactions), 8 nodes (28 interactions), 6 nodes (15 interactions), 8 nodes (28 interactions) and 6 nodes (15 interactions). The details of the P-values and nodes are listed in Table I.

Pathway enrichment analysis of the attractor modules. Further to disclose the functional enrichment of these attractor modules, pathway enrichment analysis was performed. Under the adjusted P-value $<0.05$, we found that there was no pathway enriched in module 1 and module 5. While module 2 was enriched in oxidative phosphorylation $\left(\mathrm{P}=1.63 \times 10^{-10}\right)$, module 3 was enriched in EIF2 signaling $\left(\mathrm{P}=3.67 \times 10^{-12}\right)$, module 4 was enriched in neuroactive ligand-receptor interaction $\left(\mathrm{P}=1.11 \times 10^{-3}\right)$ and module 6 was enriched in circadian rhythm $\left(\mathrm{P}=0.2 .31 \times 10^{-3}\right)$. We predicted that these attractor modules mainly influenced the pathway functions during the occurrence and development of SALS.

\section{Discussion}

In the present study, we combined the systemic module inference method with the attract method to perform analysis on the gene expression of SALS to identify the attractor modules for SALS, expecting to gain further clarification. By performing this integrated approach, we successfully identified 6 attractor modules, where module 2 was enriched in oxidative phosphorylation pathway, module 3 was enriched in EIF2 signaling pathway, module 4 was enriched in neuroactive ligand-receptor interaction pathway and module 6 was enriched in circadian rhythm pathway.

ALS is one of the most destructive neurological diseases. Worse, most of ALS patients can survival only 3-4 years after symptom onset (28). Fortunately, there are still around $10 \%$ of patients that can live beyond 10 years after symptom onset, which is mainly related to younger age of onset, pure lower motor neuron involvement, or pure upper neuron involvement (29). Researchers have focused on studying the oxidative stress in clinical or patient-oriented SALS during the last several years. Analyses of post-mortem neuronal tissue in SALS patients consistently showed that oxidation did result in damage to proteins, lipids or DNA (30). The study of the effects of oxidative stress on molecular targets and the identification of reliable biomarkers involved in oxidative stress are two major challenges in SALS (31). Although the evidence for oxidative damage in the pathogenesis of SALS is extensive, the ultimate triggers of increased levels of reactive oxygen species remain unknown, leading to speculation that oxidative stress is a major cause of the disease or is only a secondary consequence (32). In the present study, oxidative phosphorylation pathway was a pathway that one of the attractor modules identified, which exposed the close connection between the SALS and the oxidative stress.

In addition, the pathway of neuroactive ligand-receptor interaction was also shown great importance during the progress of SALS in the present study, and the module genes of SCTR, SCT, IAPP, ADCY7, GIPR and VIPR2 were enriched in this pathway. It is well known that ALS is a devastating neurological disease, hence, there may be no dispute or divergent idea that there is a close connection between the pathway of neuroactive ligand-receptor interaction with SALS. Moreover, it was indicated that gene $A D C Y 7$ presented an important role in the central nervous system, hence, $A D C Y 7$ may have properties related to cell viability and may potentially be ALS pathology (33). In addition, the pituitary adenylyl cyclase activating polypeptide (PACAP) originally isolated from the hypothalamus is a member of the vasoactive intestinal polypeptide (VIP)/mycin/glucagon superfamily (34). VPAC1 and VPAC2 bind PACAP and related neuropeptide VIPs with similar affinities are expressed by various cell types including neurons, glial cells, endothelial cells, lymphocytes, and macrophages (35). Therefore, endogenous PACAP may promote microglial destruction, and these functions are thought to drive progression of ALS disease (36). VAPC2 is produced by VIPR2 gene during the generation of gene-specific ribonucleic probes for RNA in situ hybridization experiments (36). In this case, there might be some relationship between the VIPR 2 and SALS. Verification experiments will be conducted to confirm the roles of these attractor modules on the SALS pathology.

Therefore, the approach of integrating the systemic module inference method with the attract method to perform analysis on the gene expression of SALS to identify the attractor modules for SALS was suitable. Six attractor modules (module 1 - module 6) were identified for SALS, where module 2 was enriched in oxidative phosphorylation, module 3 was enriched in EIF2 signaling, module 4 was enriched in neuroactive ligand-receptor interaction and module 6 was enriched in circadian rhythm. We predicted that these attractor modules mainly influenced these pathways to function during the occurrence and development of SALS, and these attractor modules might be potential biomarkers for early diagnosis and therapy of SALS, which could provide insight into the disease biology and suggest possible directions for drug screening programs, or even provide a hand for future study of related disease research.

\section{Acknowledgements}

Not applicable.

\section{Funding}

No funding was received.

\section{Availability of data and materials}

The datasets used and/or analyzed during the current study are available from the corresponding author on reasonable request. 


\section{Authors' contributions}

FZ conceived the study and drafted the manuscript. ML and QL acquired the data. QL and FXS analyzed the data and revised the manuscript. All authors read and approved the final manuscript.

\section{Ethics approval and consent to participate}

Not applicable.

\section{Patient consent for publication}

Not applicable.

\section{Competing interests}

The authors declare that they have no competing interests.

\section{References}

1. Carvalho TL, de Almeida LM, Lorega CM, Barata MF Ferreira ML, de Brito-Marques PR and Correia Cda C: Depression and anxiety in individuals with amyotrophic lateral sclerosis: A systematic review. Trends Psychiatry Psychother 38 $1-5,2016$

2. Leblond CS, Gan-Or Z, Spiegelman D, Laurent SB, Szuto A, Hodgkinson A, Dionne-Laporte A, Provencher P, de Carvalho M, Orrù S, et al: Replication study of MATR3 in familial and sporadic amyotrophic lateral sclerosis. Neurobiol Aging 37: 209. e17-209.e21, 2016.

3. Basu S, Baghel NS, Puri A, Shet T and Merchant NH: 18 F-FDG avid lesion due to coexistent fibrous dysplasia in a child of embryonal rhabdomyosarcoma: Source of false positive FDG-PET. J Cancer Res Ther 6: 92-94, 2010.

4. Renton AE, Chiò A and Traynor BJ: State of play in amyotrophic lateral sclerosis genetics. Nat Neurosci 17: 17-23, 2014.

5. Poppe L, Rué L, Robberecht W and Van Den Bosch L: Translating biological findings into new treatment strategies for amyotrophic lateral sclerosis (ALS). Exp Neurol 262: 138-151, 2014.

6. Ahmed RM, Irish M, Piguet O, Halliday GM, Ittner LM Farooqi S, Hodges JR and Kiernan MC: Amyotrophic lateral sclerosis and frontotemporal dementia: Distinct and overlapping changes in eating behaviour and metabolism. Lancet Neurol 15: 332-342, 2016

7. Tiloca C, Ticozzi N, Pensato V, Corrado L, Del Bo R, Bertolin C, Fenoglio C, Gagliardi S, Calini D, Lauria G, et al; SLAGEN Consortium: Screening of the PFN1 gene in sporadic amyotrophic lateral sclerosis and in frontotemporal dementia. Neurobiol Aging 34: 1517.e9-1517.e10, 2013.

8. Fogh I, Lin K, Tiloca C, Rooney J, Gellera C, Diekstra FP, Ratti A, Shatunov A, van Es MA, Proitsi P, et al: Association of a locus in the CAMTA1 gene with survival in patients with sporadic amyotrophic lateral sclerosis. JAMA Neurol 73: 812-820, 2016.

9. Lin KP, Tsai PC, Liao YC, Chen WT, Tsai CP, Soong BW and Lee YC: Mutational analysis of MATR3 in Taiwanese patients with amyotrophic lateral sclerosis. Neurobiol Aging 36: 2005.e1-4, 2015.

10. Aronica E, Baas F, Iyer A, ten Asbroek AL, Morello G and Cavallaro S: Molecular classification of amyotrophic lateral sclerosis by unsupervised clustering of gene expression in motor cortex. Neurobiol Dis 74: 359-376, 2015.

11. Jordán F, Nguyen T-P and Liu WC: Studying protein-protein interaction networks: A systems view on diseases. Brief Funct Genomics 11: 497-504, 2012.

12. Choi JK, Yu U, Yoo OJ and Kim S: Differential coexpression analysis using microarray data and its application to human cancer. Bioinformatics 21: 4348-4355, 2005.

13. Ravasz E, Somera AL, Mongru DA, Oltvai ZN and Barabási AL: Hierarchical organization of modularity in metabolic networks. Science 297: 1551-1555, 2002.

14. Srihari S and Ragan MA: Systematic tracking of dysregulated modules identifies novel genes in cancer. Bioinformatics 29: 1553-1561, 2013.
15. Mar JC, Matigian NA, Quackenbush J and Wells CA: attract: A method for identifying core pathways that define cellular phenotypes. PLoS One 6: e25445, 2011.

16. Ma L, Robinson LN and Towle HC: ChREBP*Mlx is the principal mediator of glucose-induced gene expression in the liver. J Biol Chem 281: 28721-28730, 2006.

17. Rifai N and Ridker PM: Proposed cardiovascular risk assessment algorithm using high-sensitivity $\mathrm{C}$-reactive protein and lipid screening. Clin Chem 47: 28-30, 2001

18. Pepper SD, Saunders EK, Edwards LE, Wilson CL and Miller CJ: The utility of MAS5 expression summary and detection call algorithms. BMC Bioinformatics 8: 273, 2007.

19. Giorgi FM, Bolger AM, Lohse M and Usadel B: Algorithm-driven artifacts in median polish summarization of microarray data. BMC Bioinformatics 11: 553, 2010.

20. Liu G, Wong L and Chua HN: Complex discovery from weighted PPI networks. Bioinformatics 25: 1891-1897, 2009.

21. Tavazoie SF, Alarcón C, Oskarsson T, Padua D, Wang Q, Bos PD, Gerald WL and Massagué J: Endogenous human microRNAs that suppress breast cancer metastasis. Nature 451: 147-152, 2008.

22. Tomita E, Tanaka A and Takahashi H: The worst-case time complexity for generating all maximal cliques and computational experiments. Theor Comput Sci 363: 28-42, 2006.

23. Mohseni-Zadeh S, Brézellec P and Risler J-L: Cluster-C: an algorithm for the large-scale clustering of protein sequences based on the extraction of maximal cliques. Comput Biol Chem 28: 211-208, 2004.

24. Srihari S and Leong HW: A survey of computational methods for protein complex prediction from protein interaction networks. J Bioinform Comput Biol 11: 1230002, 2013.

25. Seifoddini $\mathrm{H}$ and Djassemi M: The production data-based similarity coefficient versus Jaccard's similarity coefficient. Comput Ind Eng 21: 263-266, 1991.

26. Tian L, Greenberg SA, Kong SW, Altschuler J, Kohane IS and Park PJ: Discovering statistically significant pathways in expression profiling studies. Proc Natl Acad Sci USA 102: 13544-13549, 2005.

27. Benjamini Y and Hochberg Y: Controlling The false discovery rate - A practical and powerful approach to multiple testing. J R Stat Soc Ser A Stat Soc 57: 289-300, 1995.

28. Yokoi D, Atsuta N, Watanabe H, Nakamura R, Hirakawa A, Ito M, Watanabe $\mathrm{H}$, Katsuno M, Izumi Y, Morita M, et al; JaCALS: Age of onset differentially influences the progression of regional dysfunction in sporadic amyotrophic lateral sclerosis. J Neurol 263: 1129-1136, 2016.

29. Allen SP, Rajan S, Duffy L, Mortiboys H, Higginbottom A, Grierson AJ and Shaw PJ: Superoxide dismutase 1 mutation in a cellular model of amyotrophic lateral sclerosis shifts energy generation from oxidative phosphorylation to glycolysis. Neurobiol Aging 35: 1499-1509, 2014.

30. Calingasan NY, Chen J, Kiaei M and Beal MF: Beta-amyloid 42 accumulation in the lumbar spinal cord motor neurons of amyotrophic lateral sclerosis patients. Neurobiol Dis 19: 340-347, 2005.

31. Bogdanov M, Brown RH Jr, Matson W, Smart R, Hayden D, O'Donnell H, Flint Beal $\mathrm{M}$ and Cudkowicz $\mathrm{M}$ : Increased oxidative damage to DNA in ALS patients. Free Radic Biol Med 29: 652-658, 2000.

32. D'Amico E, Factor-Litvak P, Santella RM and Mitsumoto H: Clinical perspective on oxidative stress in sporadic amyotrophic lateral sclerosis. Free Radic Biol Med 65: 509-527, 2013.

33. Abalkhail H, Mitchell J, Habgood J, Orrell R and de Belleroche J: A new familial amyotrophic lateral sclerosis locus on chromosome 16q12.1-16q12.2. Am J Hum Genet 73: 383-389, 2003.

34. Miyata A, Arimura A, Dahl RR, Minamino N, Uehara A, Jiang L, Culler MD and Coy DH: Isolation of a novel 38 residuehypothalamic polypeptide which stimulates adenylate cyclase in pituitary cells. Biochem Biophys Res Commun 164: 567-574, 1989.

35. Dickson L and Finlayson K: VPAC and PAC receptors: From ligands to function. Pharmacol Ther 121: 294-316, 2009.

36. Ringer C, Büning LS, Schäfer MKH, Eiden LE, Weihe E and Schütz B: PACAP signaling exerts opposing effects on neuroprotection and neuroinflammation during disease progression in the SOD1(G93A) mouse model of amyotrophic lateral sclerosis. Neurobiol Dis 54: 32-42, 2013.

This work is licensed under a Creative Commons Attribution-NonCommercial-NoDerivatives 4.0 International (CC BY-NC-ND 4.0) License. 